\title{
Instrument Retrieval from Mandibular Second Molar Using Masserann Technique and Management of an Iatrogenic Defect Caused During Retrieval by Treating It as A Canal Extension: A Case Report
}

\author{
A. R. Vivekananda Pai
}

Department of Conservative Dentistry and Endodontics, Melaka Manipal Medical College (Manipal Academy of Higher Education)(India)

\section{ABSTRACT}

Masserann technique is one of the methods suggested for retrieval of separated instruments. However, its application in posterior teeth is risky, difficult, and clinically challenging. This case report illustrates successful application of Masserann technique to retrieve a separated instrument from the middle third of a mesial canal of a mandibular second molar and management of an iatrogenic defect, which was caused during retrieval, by treating it as a canal extension during obturation. This obturation was done with glass ionomer cement based sealer for canal reinforcement. In the absence of other effective techniques, when the separated instrument is tightly lodged in the canal, Masserann technique could be useful for instrument retrieval in posterior teeth. However, its success depends on case selection and clinical skill. Since this technique leads to removal of considerable amount of dentin, use of bonding or adhesive obturation materials could be considered to reinforce and strengthen the root.

\section{Keywords: Canal Reinforcement, Instrument Retrieval, Mandibular Second Molar, Masserann Technique,} Orthograde Approach, Separated Instrument

\section{Introduction}

Masserann technique is one of the methods employed for orthograde retrieval of separated instruments, silver points and posts from the root canal. It is more effective in anterior teeth with a higher success rate. However, in posterior teeth this technique is risky and has limited application with lower success rate due to restricted accessibility, less root bulk and the presence of canal curvatures, particularly when employed at or apical to mid root level. [1-5] Masserann technique can lead to iatrogenic errors such as excessive removal of dentin, root weakening, and root perforation. [5, 6] Nevertheless, Masserann technique is effective in retrieval of separated instruments because of its locking and gripping mechanism though its application in selected posterior teeth is a difficult and a clinically challenging task. [1, 3]

This case report illustrates successful application of Masserann technique to retrieve a separated instrument from the middle third of a mesial canal of a mandibular second molar and management of an iatrogenic defect by treating it as a canal extension during obturation which was done with a glass ionomer cement based sealer for canal reinforcement.

\section{Case Report}

A 25-year-old female patient undergoing endodontic treatment in the mandibular right second molar tooth (tooth 47) was presented with a separated instrument in the mesio-lingual canal. The separated instrument was a size 25 , stainless steel K-file. Periapical radiographic examination of tooth 47 showed that the separated fragment was approximately $3 \mathrm{~mm}$ in length and was lodged in the middle third of the root canal which displayed a moderate curvature (Fig 1). Bypassing the fragment with size 08 and size $10 \mathrm{~K}$-files (Dentsply Maillefer, Ballaigues, Switzerland) failed as the fragment was tightly lodged in the canal. Due to the lack of accessibility to instruments such as ultrasonics or other techniques, a decision was taken to retrieve the fragment using a readily available Masserann kit (Micro Mega, Besancon, France). The treatment was carried out without rubber dam isolation as patient was asthmatic with breathing difficulties and objected to rubber dam placement.

The working length from occlusal reference point up to the fragment was noted. Radicular access to the fragment was straightened using Gates-Glidden drills (Mani inc., Japan). An end cutting trephan with a diameter of $1.2 \mathrm{~mm}$ was run at a slow speed in an anticlockwise direction to cut 
the root dentin around the fragment. The apical progress of the trephan was monitored with radiographs (Fig 2). Approximately $1.5 \mathrm{~mm}$ of dentin surrounding the fragment was removed to prepare a trough around coronal end of the fragment. An extractor tube of $1.2 \mathrm{~mm}$ diameter was gently inserted into the trough and plunger rod of the tube was rotated in a clockwise direction to wedge and grip the fragment against the wall of the extractor tube. The extractor tube gripping the fragment was confirmed with a radiograph (Fig 3) and gently turned in an anti-clockwise direction to dislodge and retrieve the fragment from the canal. Retrieval of the fragment was radiographically confirmed (Fig 4).

A ledge was detected apical to the site of retrieval. similarly, although no perforation was noticed, a deep recess-like gouged defect was detected on the mesial aspect of the canal apical to the site of retrieval. This defect probably occurred during troughing which was carried out more at the expense of mesial wall of the canal. The ledge was negotiated and canal cleaning and shaping was completed. Later, obturation was carried out using gutta percha cones, glass ionomer cement based sealer Ketac-Endo (ESPE, GBMH \& Co., Seefeld-Oberbay, Germany) and lateral compaction technique. In the instrument retrieved canal, sealer was applied liberally and obturation was carried out gently to minimize sealer backflow from the orifice and have more sealer mass, particularly coronal to the site of retrieval, for canal reinforcement and strengthening of the root. The gouged defect on the mesial aspect was treated as a canal extension and obturated with the main canal. The access opening was sealed and the patient was scheduled for post endodontic restoration (Fig 5).

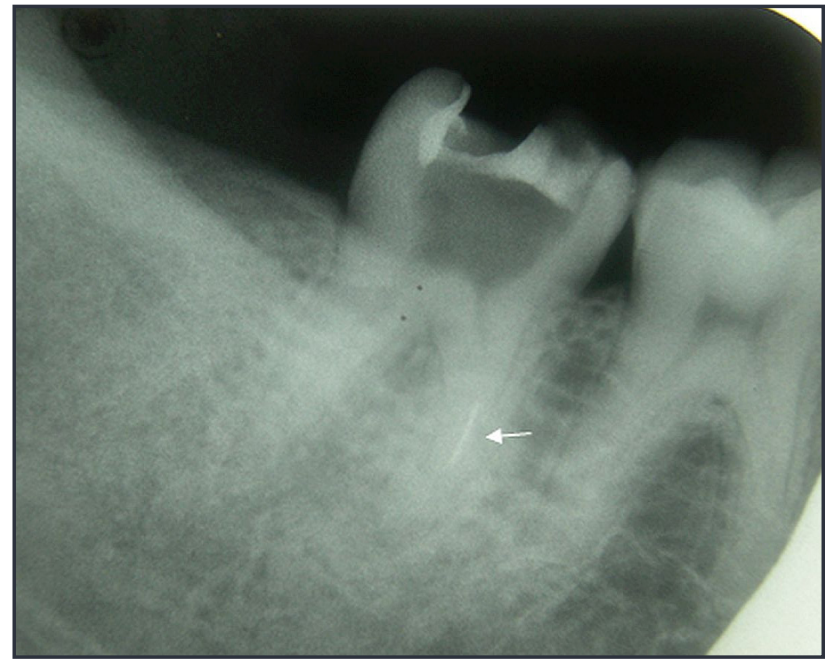

Fig. 1: Preoperative periapical radiograph showing a separated instrument (white arrow) in the middle third of mesio-lingual canal of tooth 47.

\section{Discussion}

Application of Masserann technique in a mandibular molar, particularly in a mesial canal, is difficult due to posterior location of tooth with difficulty in obtaining and operating along a straight line access and limited visibility. Additionally, this technique is more difficult in the mesial canal of mandibular second and third molars because of limited space to operate along a straight line access. This technique is also risky in the mesial canal of a mandibular molar due to crown and root anatomy and angulation, limited thickness of canal dentine, varying thickness of dentine on mesial and distal aspects of canal, and canal curvature. $[6,7]$ Trephanation in a mesial canal, particularly in the middle and apical portions, of a mandibular molar has the risk of thinning and weakening or perforation of the root. $[3,5,6]$ However, these risks could be minimized by combining Masserann technique with ultrasonics, surgical operating microscope, or fibre-optic illumination. $[3,6]$ The success of Masserann technique depends on case selection and is influenced by type of tooth, root thickness, canal curvature, location of the separated instrument, and accessibility. It also depends on the skill, experience and patience of the operator. [1-3]

Masserann technique leads to removal of considerable amount of dentin and may weaken the tooth with a tendency to vertical root fracture. [5, 6] Therefore, following application of this technique, using any bonding or adhesive material in the canal could be considered clinically beneficial. In the present case, a glass ionomer cement based sealer was used in order to strengthen the root. This sealer shows adhesion to dentine by means of

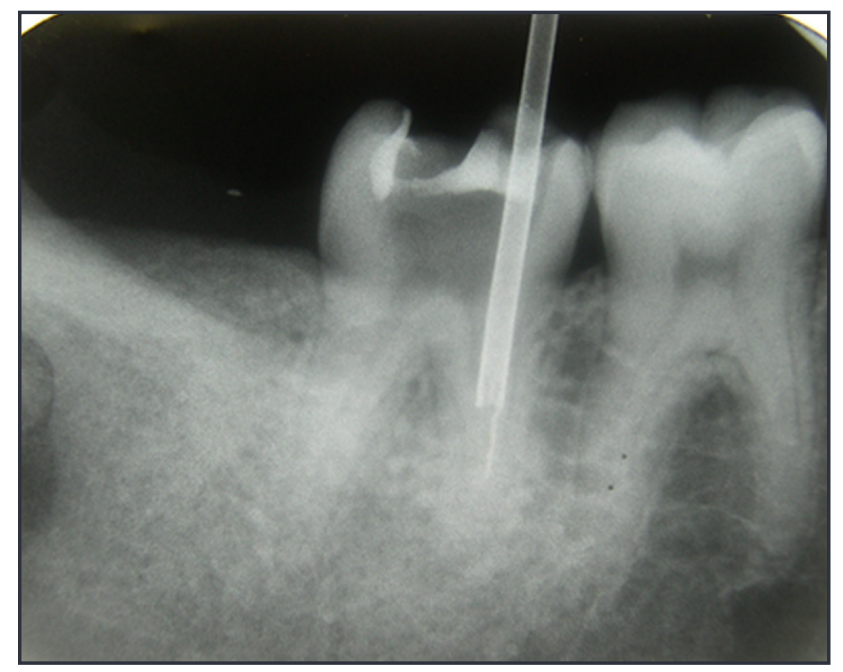

Fig. 2: Periapical radiograph showed the position of the trephan in relation to the separated instrument in tooth 47. 


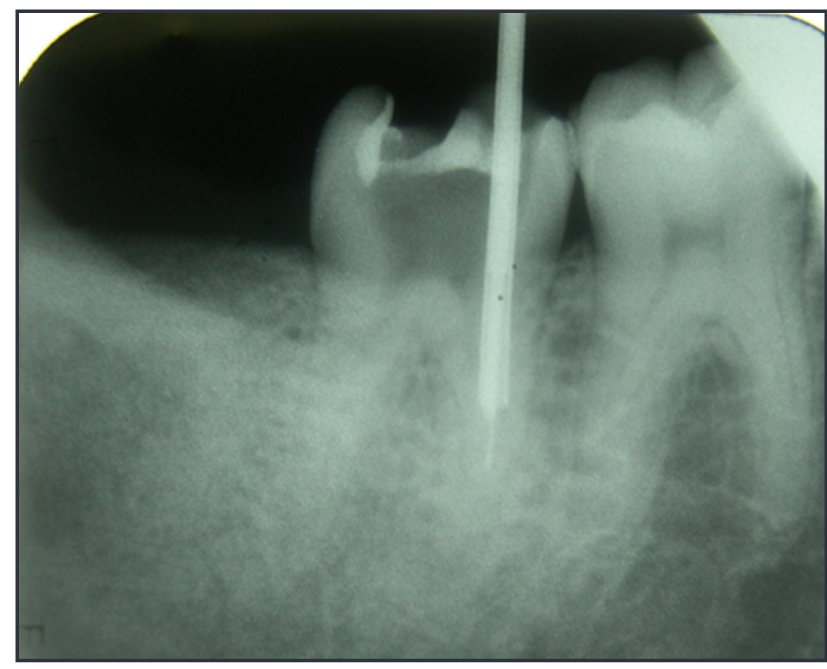

Fig. 3: Periapical radiograph confirmed gripping of the separated instrument by the extractor tube in tooth 47 .

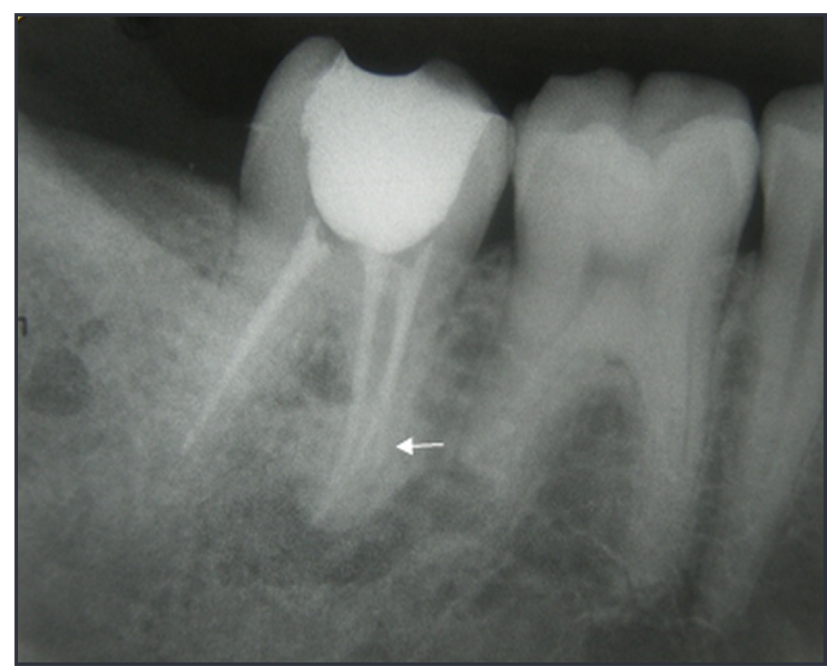

Fig. 5: Post-obturation periapical radiograph of tooth 47. Note the iatrogenic defect on the mesial aspect of instrument retrieved canal treated as a canal extension and obturated (white arrow) with the main canal.

chemical bonding and strengthens an endodontically treated tooth. [8] It is shown to reinforce weakened roots and make them less susceptible to vertical root fracture. $[9,10]$

\section{Conclusion}

This case report emphasizes that Masserann technique, in the absence of other effective techniques, can be useful to retrieve a tightly lodged separated instrument from the middle third of mesial canal of a mandibular molar. However, its success depends on proper case selection with good bulk of dentine, less canal curvature and straight line access and superior clinical skill. Since this technique

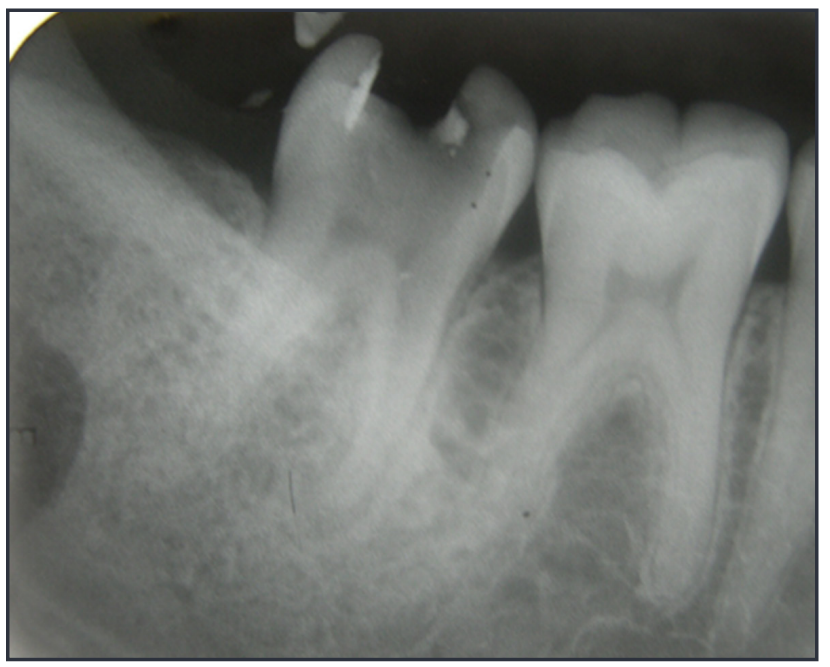

Fig. 4: Periapical radiograph confirmed the successful retrieval of the separated instrument from tooth 47 .

leads to removal of considerable amount of dentin, use of bonding or adhesive obturation materials could be considered to reinforce and strengthen the root.

\section{Acknowledgements}

The author expresses his sincere gratitude to Late Dr. M. Premanand Kamath (Ex-faculty and former Professor and Head, Department of Conservative Dentistry and Endodontics, Manipal College of Dental Sciences [Manipal Academy of Higher Education], Mangalore, Karnataka, India) for his valuable guidance and support while carrying out the clinical procedures reported in this manuscript.

\section{Reference}

1. Freidman S, Stabholz A, Tamse A. Endodontic retreatment: case selection and techniques. Part 3. Retreatment techniques. J Endod 1990; 16: 543-9.

2. Hulsmann M. Methods for removing metal obstruction from the root canal. Endod Dent Traumatol 1993;9: 223-37.

3. Okiji T. Modified usage of the Masserann kit for removing intracanal broken instruments. J Endod 2003; 29: 466-7.

4. Gencoglu N, Helvacioglu D. Comparison of the different techniques to remove fractured endodontic instruments from root canal systems. Eur J Dent 2009; 3: 90-5.

5. Terauchi Y, O'Leary L, Suda H. Removal of separated files from root canals with a new file removal system: case reports. J Endod 2006; 32: 789-97.

6. Yoldas O, Oztunc H, Tinaz C, Alparslan N. Perforation risks associated with the use of Masserann endodontic kit drills in mandibular molars. Oral Surg Oral Med Oral Pathol Oral Radiol Endod 2004; 97: 513-7.

7. Scheid RC, Weiss G. Woelfel's Dental Anatomy. 8th ed. Philadelphia: Lippincoat Williams \& Wilkins; 2012. 
8. Timpawat S, Harnirattisai C, Senawongs P. Adhesion of a glass-ionomer root canal sealer to the root canal wall. $\mathrm{J}$ Endod 2001; 27: 168-71.

9. Cobankara FK, Ungör M, Belli S. The effect of two different root canal sealers and smear layer on resistance to root fracture. J Endod 2002; 28: 606-9.

10. Lertchirakarn V, Timyam A, Messer HH. Effects of root canal sealers on vertical root fracture resistance of endodontically treated teeth. J Endod 2002; 28: 217-9.

\section{*Corresponding author:}

Dr. A. R. Vivekananda Pai, Professor and Head, Department of Conservative Dentistry and Endodontics, Faculty of Dentistry, Melaka Manipal Medical College (Manipal Academy of Higher Education), Jalan Batu Hampar, Bukit Baru, 75150 Melaka, Malaysia.

Phone: +91 (606) 289 6662, Ext: 3820

Email: drpaivivekananda@gmail.com

Financial or other Competing Interests: None. 